\title{
Whole Exome Sequencing Uncovers Germline Variants of Cancer-Related Genes in Sporadic Pheochromocytoma
}

\author{
Milena Urbini $\left(\mathbb{D},{ }^{1}\right.$ Margherita Nannini $\left(\mathbb{D},{ }^{2}\right.$ Annalisa Astolfi $\left(\mathbb{D},{ }^{1}\right.$ Valentina Indio, ${ }^{1}$ \\ Valentina Vicennati, ${ }^{3}$ Matilde De Luca, ${ }^{1}$ Giuseppe Tarantino, ${ }^{1}$ Federica Corso, ${ }^{2}$ \\ Maristella Saponara, ${ }^{2}$ Lidia Gatto, ${ }^{2}$ Donatella Santini, ${ }^{4}$ Guido Di Dalmazi, ${ }^{3}$ Uberto Pagotto, ${ }^{3}$ \\ Renato Pasquali, ${ }^{3}$ Andrea Pession, ${ }^{2}$ Guido Biasco, ${ }^{1,2}$ and Maria A. Pantaleo ${ }^{1,2}$ \\ 1 "Giorgio Prodi" Cancer Research Center, University of Bologna, Bologna, Italy \\ ${ }^{2}$ Department of Specialized, Experimental and Diagnostic Medicine, S. Orsola-Malpighi Hospital, University of Bologna, \\ Bologna, Italy \\ ${ }^{3}$ Endocrinology Unit, Department of Medical and Surgical Sciences, Center for Applied Biomedical Research, \\ S. Orsola-Malpighi Hospital, University of Bologna, Bologna, Italy \\ ${ }^{4}$ Pathology Unit, S. Orsola-Malpighi Hospital, University of Bologna, Bologna, Italy
}

Correspondence should be addressed to Margherita Nannini; margherita.nannini@unibo.it

Received 6 February 2018; Revised 8 May 2018; Accepted 29 May 2018; Published 19 August 2018

Academic Editor: Atsushi Kurabayashi

Copyright (C) 2018 Milena Urbini et al. This is an open access article distributed under the Creative Commons Attribution License, which permits unrestricted use, distribution, and reproduction in any medium, provided the original work is properly cited.

Background. Pheochromocytomas (PCCs) show the highest degree of heritability in human neoplasms. However, despite the wide number of alterations until now reported in PCCs, it is likely that other susceptibility genes remain still unknown, especially for those PCCs not clearly syndromic. Methods. Whole exome sequencing of tumor DNA was performed on a set of twelve PCCs clinically defined as sporadic. Results. About $50 \%$ of PCCs examined had somatic mutations on the known susceptibility $V H L$, $N F 1$, and RET genes. In addition to these driver events, mutations on SYNE1, ABCC10, and RAD54B genes were also detected. Moreover, extremely rare germline variants were present in half of the sporadic PCC samples analyzed, in particular variants of MAX and SAMD9L were detected in the germline of cases wild-type for mutations in the known susceptibility genes. Conclusions. Additional somatic passenger mutations can be associated with known susceptibility VHL, NF1, and RET genes in PCCs, and a wide number of germline variants with still unknown clinical significance can be detected in these patients. Therefore, many efforts should be aimed to better define the pathogenetic role of all these germline variants for discovering novel potential therapeutic targets for this disease still orphan of effective treatments.

\section{Introduction}

Pheochromocytomas (PCCs) are rare tumors of the autonomic nervous system that arise from the chromaffin tissue of the adrenal medulla [1]. Most of PCCs are benign; however, approximately $10 \%$ of cases are malignant and can develop metastases either at the time of diagnosis or even later after several years, with a highly variable clinical course and a 5 -year overall survival rate of 50\% [2]. PCCs show the highest degree of heritability in human neoplasms and almost $40 \%$ of cases occur within heritable syndromes, including multiple endocrine neoplasia type 2 (MEN2), neurofibromatosis type 1 (NF1), von Hippel Lindau (VHL) disease, and hereditary paraganglioma and familial pheochromocytoma [3]. Germline mutations have been identified in more than 15 well-characterized genes, such as VHL, SDHB, SDHD, NF1, and RET [4].

Despite this well-known inherited basis of PCCs and paragangliomas (PGLs), during the past decades somatic mutations with variable frequency in many genes, including EPAS1 (HIF2 $\alpha$ ), RET, VHL, RAS, NF1, ATRX, and CSDE1 recurrent somatic copy number alterations and several fusion genes, involving MAML3, BRAF, NGFR, and NF1, have been progressively identified [5-16]. Recently a multiplatform 
TABle 1: Patient characteristics.

\begin{tabular}{|c|c|c|c|c|c|}
\hline ID & Sex & Age & Tumor size $(\mathrm{cm})$ & HIC characteristics & PASS score \\
\hline N47 & $\mathrm{F}$ & 63 & 5.0 & Ki-67 2.4\% & n.a. \\
\hline N49 & M & 65 & 4.0 & Positive staining for synaptophysin. Ki-67 3.2\% & 3 \\
\hline N50 & M & 36 & 4.0 & $\begin{array}{l}\text { Positive staining for synaptophysin. S100 protein-positive sustentacular cells. } \\
\qquad \text { Ki-67 2.2\% }\end{array}$ & 3 \\
\hline N51 & M & 46 & 1.5 & $\begin{array}{l}\text { Positive staining for chromogranin A and synaptophysin. S100 protein- } \\
\text { positive sustentacular cells. Negative staining for c-kit, EGFr, p53. Ki-67 0.5\% }\end{array}$ & 2 \\
\hline N52 & M & 47 & 3.5 & $\begin{array}{l}\text { Positive staining for chromogranin A and synaptophysin. S100 protein- } \\
\text { positive sustentacular cells. Negative staining for CD10. Ki-67 0.1\% }\end{array}$ & 4 \\
\hline N53 & $\mathrm{F}$ & 58 & 5.4 & Ki-67 1.8\% & 5 \\
\hline N54 & $\mathrm{F}$ & 46 & 6.0 & $\begin{array}{l}\text { Positive staining for chromogranin A and synaptophysin. S100 protein- } \\
\text { positive sustentacular cells. Focal positive staining for CD10. Ki-67 0.4\% }\end{array}$ & 6 \\
\hline N55 & $\mathrm{F}$ & 30 & 6.0 & $\begin{array}{l}\text { Positive staining for chromogranin A and synaptophysin. S100 protein- } \\
\text { positive sustentacular cells. Ki-67 0.1\% }\end{array}$ & 4 \\
\hline N56 & M & 41 & 1.7 & $\begin{array}{l}\text { Positive staining for synaptophysin. S100 protein-positive sustentacular cells. } \\
\text { Negative staining for calretinin. Ki-67 } 2.2 \%\end{array}$ & 2 \\
\hline N57 & M & 35 & 8.0 & $\begin{array}{l}\text { Positive staining for chromogranin A and synaptophysin. Few S100 protein- } \\
\text { positive sustentacular cells. Negative staining for calretinin and } \alpha \text {-inhibin. } \\
\text { Ki-67 5.7\% }\end{array}$ & 4 \\
\hline N62 & M & 59 & 3.0 & Ki-67 1.4\%. & 5 \\
\hline N63 & M & 26 & 7.0 & $\begin{array}{l}\text { Positive staining for synaptophysin. S100 protein-positive sustentacular cells. } \\
\text { Negative staining for calretinin, MEL-A, and } \alpha \text {-inhibin. Ki- } 674 \%\end{array}$ & 14 \\
\hline
\end{tabular}

integrated analysis classified PCCs/PGLs into four clinically relevant molecular subtypes: a kinase signaling subtype, a pseudohypoxia subtype, a Wnt-altered subtype driven by $M A M L 3$ and CSDE1, and a cortical admixture subtype [16].

Therefore, given this molecular complexity of PCCs, it is likely that other alterations remain still unknown, especially for those PCCs not clearly syndromic. For this purpose, in the present study, we performed whole exome sequencing on a set of 12 clinically sporadic PCCs, with a family history negative for PCCs/PGLs.

\section{Materials and Methods}

2.1. Patients and Tumor Samples. Fresh tissue specimens of PCC from 12 patients with a family history negative for PCCs/PGLs were collected during the surgical operation, snap-frozen in liquid nitrogen, and stored at $-80^{\circ} \mathrm{C}$ until analysis. Patient characteristics are listed in Table 1 . Whole exome sequencing was performed on biological tumor samples on matched peripheral blood samples obtained from all patients. This study was approved by the local institutional ethical committee of S. Orsola-Malpighi hospital (approval number 95/2013/U/Tess). All patients provided written informed consent.

2.2. Whole Exome Sequencing. DNA was extracted from peripheral blood and fresh frozen tissue with DNA mini kit (Qiagen, Milan, Italy) following manufacturer's instructions. Whole exome sequencing of tumor DNA was performed on HiScanSQ platform in accordance with Nextera Rapid Exome Enrichment protocol (Illumina, San Diego, California, USA). Briefly, $100 \mathrm{ng}$ of genomic DNA was tagged and fragmented by the Nextera transposome. The Nextera transposome simultaneously fragments the genomic DNA and adds adapter sequences to the ends. The products were then amplified and exome regions were enriched. The enriched libraries were amplified by PCR and quantified using PicoGreen assay (Life Technologies, Milan, Italy).

Paired-end libraries were sequenced at $2 \times 100 \mathrm{bp}$ read length using Illumina Sequencing by synthesis (SBS) technology.

2.3. Bioinformatic Analysis. After demultiplexing and FASTQ generation performed with bcltofastq function developed by Illumina, the paired-end reads were trimmed using AdapterRemoval (https://github.com/MikkelSchubert/ adapterremoval) with the aim of removing stretches of lowquality bases $(<\mathrm{Q} 10)$ and Truseq/Nextera rapid capture adapters present in the sequences. The paired-end reads were then aligned on human reference genome hg38 (http://www. genome.ucsc.edu). Data from WES were mapped with Burrows-Wheeler Aligner with the default setting; the PCR and optical duplicates were removed, and Genome Analysis Toolkit (https://software.broadinstitute.org/gatk) was used to locally realign, recalibrate, and call the Ins/del variants, while point mutations were identified with the algorithm MuTect (https://www.broadinstitute.org/cancer/ $\mathrm{cga} /$ mutect). Single nucleotide variants (SNV) and ins/del were annotated with gene and protein alteration using Annovar (http://annovar.openbioinformatics.org); nonsynonymous and nonsense SNV, frameshift/nonframeshift Indels, and splice site mutations were selected with a threshold read depth $\geq 10 \mathrm{x}$ and a variant allele frequency $\geq 0.2$. All the variants were filtered to select novel or rare events basing on database of human variability 
dbSNP (http://www.ncbi.nlm.nih.gov/SNP), 1000Genomes (http://www.1000genomes.org), ExAC (http://exac.broad institute.org), and EVS (http://evs.gs.washington.edu/EVS). In-depth evaluation of high confidence somatic variants was performed by verifying the presence of alternate allele on the normal counterpart and manually visualizing each variation with the tview function of SAMmtools. Both somatic mutations and germline variants were searched in COSMIC (Catalog of Somatic Mutations in Cancer; http://cancer. sanger.ac.uk/cosmic), ClinVar (https://www.ncbi.nlm.nih. gov/clinvar), and HGMD (http://www.hgmd.org), and their effect on protein structure and function was predicted with SNPeff, a software that uses three different prediction algorithms (SIFT, Polyphen2, and LRT).

Moreover, based on WES data, the analysis of amplifications and large deletions were performed making a consensus between Control FREEC (http://boevalab.com/FREEC) and ADTEX (http://adtex.sourceforge.net) with paired tumour/ matched normal samples. A filtering procedure was applied taking into account the uncertainty value given by Control FREEC $(<80 \%)$ and the polymorphic copy number variants from the Database of human Genomic Variants (http://dgv. tcag.ca/dgv/app/home).

For germline variants prioritization, all rare $(\mathrm{MAF}<0.01)$ alterations occurring on the known susceptibility genes of PCC and PGL were considered. Moreover, variants with an evident effect on the protein (nonsense and splicing mutations or frameshift ins/del) were prioritized and manually annotated using HGMD and ClinVar database and with literature.

2.4. Sanger Sequencing. Sequencing of the DNA extracted from tumors and matched peripheral blood samples was performed to validate candidate mutations. Specific PCR assay for the amplification and sequencing of selected genes was designed with Primer Express 3.0 Software (Applied Biosystems, Monza, Italy). PCR products were purified with the Qiaquick PCR purification kit (Qiagen) and sequenced on both strands using the Big Dye Terminator v1.1 Cycle Sequencing kit (Applied Biosystems). Sanger Sequencing was performed on ABI 3730 Genetic Analyzer (Applied Biosystems).

2.5. Real-Time PCR. Total RNA was extracted from fresh frozen tissues using the RNeasy spin-column method (Qiagen). RNA was reverse transcribed to cDNA using the Transcriptor First-Strand cDNA Synthesis Kit (Life Technologies) with oligo dT primers. qPCR amplification of genes of interest was performed with real-time LightCycler 480 instrument (Roche). Fold-change was estimated by DDCt method, using ATPS, HPRT, and HMBS genes as housekeeping controls. Primers used were: MAX_FW $5^{\prime}$ - GCGATAACGATGAC ATCGAGGT-3' and MAX_RV $5^{\prime}$-CCCGCAAACTGTGA AAGCTGT-3', SAMD9L_FW 5' $5^{\prime}$-AAAGTGAGTGAGTG AGCCCAG-3' and SAMD9L_RV $5^{\prime}$-CATGCTCTTTGGTC CAGTCT-3', ATPS_FW 5'-GTCTTCACAGGTCATATGG GGA-3' and ATPS_RV 5'-ATGGGTCCCACCATATAGA AGG-3', HMBS_FW 5'-GGCAATGCGGCTGCAA-3' and HMBS_RV $5^{\prime}$-GGGTACCCACGGAATCAC-3', HPRT_FW $5^{\prime}$-TGACACTGGCAAAACAATGCA- $3^{\prime}$ and HPRT_RV
$5^{\prime}$-GGTCCTTTTCACCAGCAAGCT-3'. For detection of cortical admixture profile, expression levels of STAR, CYP2W1, CYP11B2, CYP21A2, and CLND2, genes were evaluated using the following primers: STAR_Fw $5^{\prime}$-TGGGCA TCCTTAGCAACCAA- $3^{\prime}$ and STAR_Rev $5^{\prime}$-GCCCACATC TGGGACCACTT-3'; CYP2W1_Fw $5^{\prime}$-GTCATGGTCCT CTTGGGGTC-3' and CYP2W1_Rev $5^{\prime}$-CTCCAGGAGGG TCCTCAGAA-3 ${ }^{\prime}$; CYP11B2_Fw 5'-TGCATCCCTGCAGG ATGAT-3' and CYP11B2_Rev $5^{\prime}$-GCGACAGCACATCT GGGT-3'; CYP21A2_Fw 5'-AGCCCGACCTCCCCAT-3' and CYP21A2_Rev $5^{\prime}$-CACCACCACATCTTGCAGCC-3'; CLND2_Fw 5'-CCCCTTGTACTTCGCTCCCC- $3^{\prime}$ and CLND2_Rev 5'-AAGCAGCCTCAAGAAGGCATC-3'.

\section{Results}

Exome sequencing generated a minimum of 45.5 million reads/sample with a mean coverage of RefSeq regions of $44 \mathrm{x}$. Few somatic mutations were identified (an average of 7 mutations per sample) while several copy number alterations were detected, with losses of chr1, chr3, and chr17 being the most recurrent.

3.1. Analysis of Somatic Mutations. The biological effect of somatic mutations was predicted with three bioinformatic tools (Suppl. Table 1). Mutations on susceptibility genes were detected in 6 out of 12 cases and were annotated in COSMIC and HGMD databases. VHL missense mutations were identified in two cases: a p.S65A identified in N51, a mutation already reported in PCC (COSM144970), and a p.Y98H in N56, a mutation reported in ClinVar and HMGD as pathogenic in association with Von Hippel-Lindau syndrome. Two novel somatic alterations were detected in NF1: N62 carried a splicing mutation c.480-1G $>\mathrm{C}$ in exon 5 and N55 had a frameshift deletion (p.W784fs) in exon 20. Interestingly, the abovementioned mutations on NF1 and VHL were in regions affected by loss of the wild-type allele (Figure 1). Heterozygous loss of the chromosomal region covering NF1 was detected also in N54; however, no additional mutational event was detected on this gene. RET was found mutated in heterozygosis in two other tumors: a novel exon 11 nonframeshift INDEL (p.L633delinsLCR) was detected in N57 and the hotspot mutation p.M918T (COSM965) in N53.

In addition to these driver mutations on known susceptibility genes, other somatic passenger mutations were detected in these 6 tumors. In particular, SYNE1 and $A B C C 10$ were mutated, respectively, in the two RET-mutated cases (N53 and N57), while a missense p.G460S mutation of $R A D 54 B$, a gene involved in DNA repair process, affected a NF1 mutated case (N55). On the other side, few somatic mutations were identified in the 6 remaining PCC cases and none was recurrent between samples. N63 carried a p.L114X nonsense mutation in CDC14B, a protein phosphatase involved in DNA damage response. N52 carried two heterozygous missense mutations (SMARCC2 p.P1092R and PRKG1 p.F387) both predicted as pathogenic by bioinformatic predictors. 


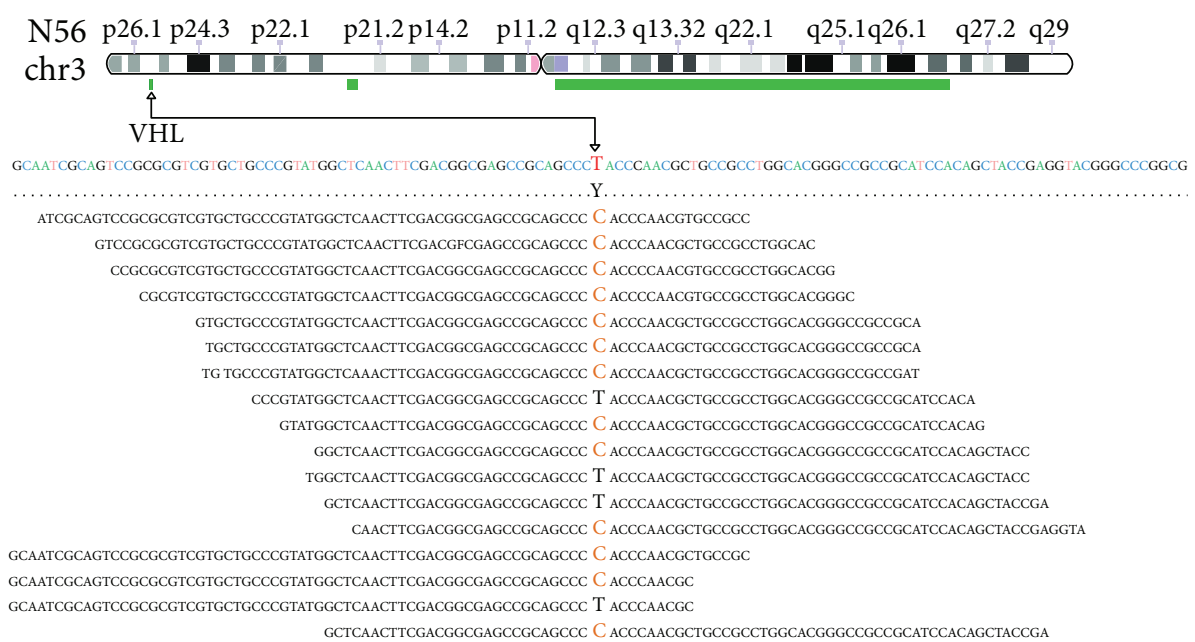

(a)

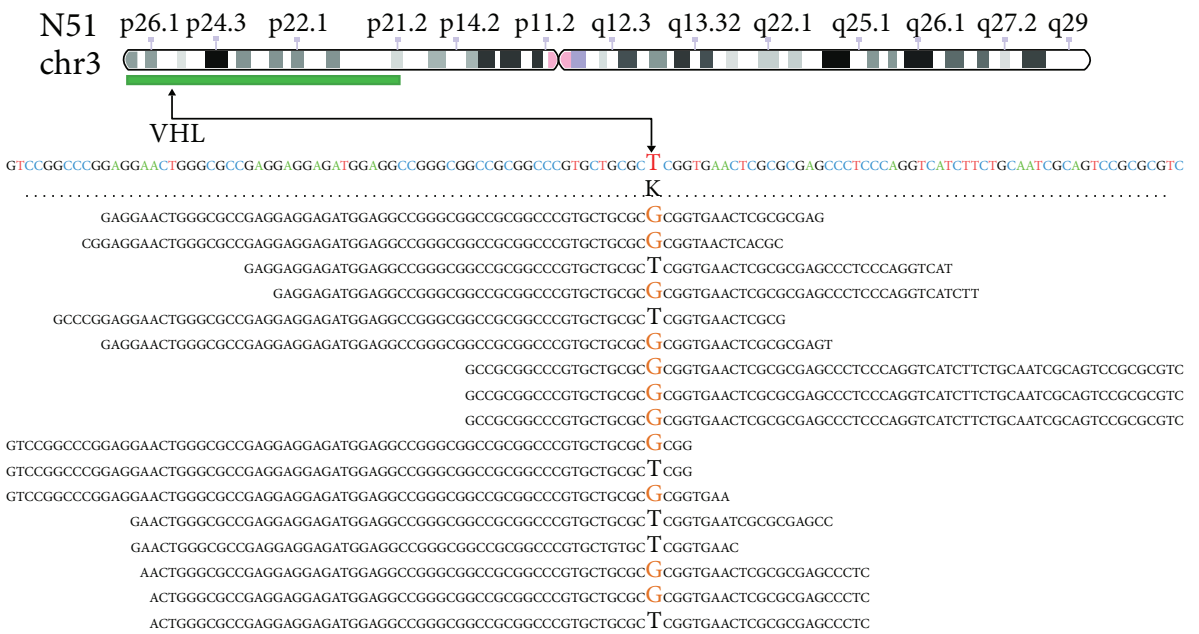

(b)

N62 p13.3 p13.2 p12 p11.2 p11.1 q11.2 q21.1 q21.31 q22 q23.2 q24.2 $\quad$ q25.2

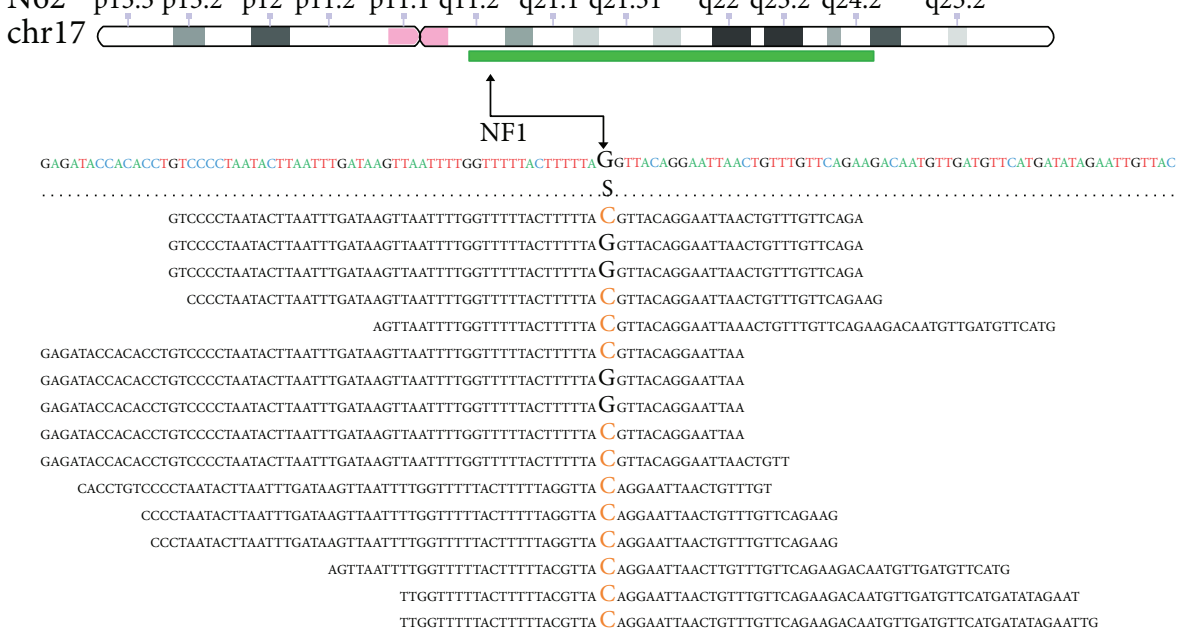

(c)

Figure 1: Continued. 


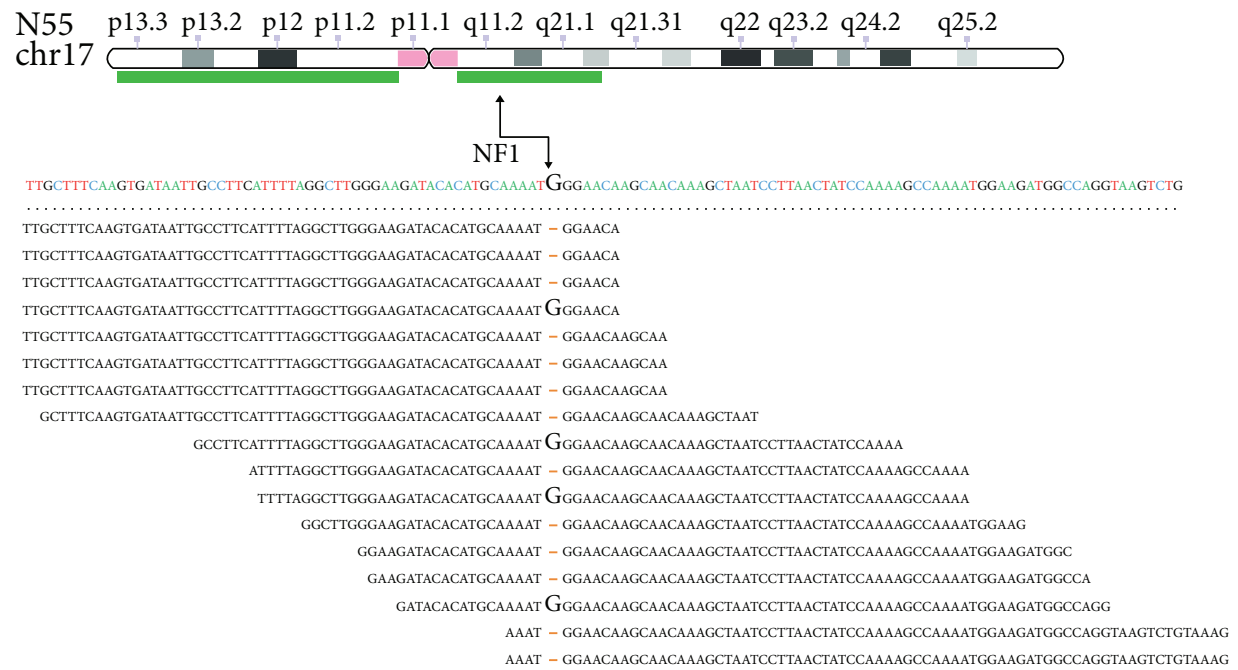

(d)

FIGURE 1: Deletion of the wild-type allele occurred in PCC samples that carry NF1 or VHL somatic mutations. Alignments of sequencing reads located on NF1 or VHL mutated bases are shown. Green bars represent deleted regions of chr3 for VHL (a, b) and chr17 for NF1 (c, d) detected by copy number analysis. A black arrow indicates the chromosome position of NF1 or VHL and the mutated base on the sequencing reads.

3.2. Analysis of Germline Rare Variants. In addition to the abovementioned somatic alterations, rare $(\mathrm{ExAc}<0.01)$ germline variants were identified in five sporadic PCC samples (Suppl. Table 2), among which those occurring in $M A X$ and $S A M D 9 L$ seem to play an important role in PCC pathogenesis, completing the picture of relevant alterations identified in our cohort (Table 2). In N63, a novel germline variant of $M A X$ (c.397-2A>G), affecting splice site and pathogenic for the protein function, was detected. Noticeably, this patient is a young adult (age 26) and showed the loss of the wild-type allele in the tumor, thus following the Knudson two-hit model (Figure 2(a)), and mRNA level of MAX was found downregulated with respect to the other PCC samples (Figure 2(b)). According to the findings of Fishbein et al. [16], we evaluated the expression level of STAR, CYP11B2, CYP2W1, CYP21A2, and CLND2 with the aim to assess whether a cortical admixture profile was present in this MAX mutated sample. However, a general low expression level of these genes was found in our cohort and no difference between the MAX mutated case and the other PCC samples was detected (data not shown). Conversely, 2 germline variants of SAMD9L were found in other 2 PCC, both wildtype for mutations in known susceptibility genes: a novel p.N769fs frameshift deletion in N47 and a rare $(\mathrm{ExAc}=0.2 \%)$ nonsense p.R406X in N50 (Figure 2(c)). Evaluation of mRNA level of SAMD9L showed a significant downregulation of the transcript in this two PCC samples (Figure 2(d)). Interestingly, the same gene was found somatically mutated in N54 (p.L1016S), increasing to 3 the number of PCC wild-type cases of our cohort that carried alterations on SAMD9L (Table 2).

On the contrary, the role of the remaining germline variants (suppl. Table 2) is not clear. In N50, in addition to SAMD9L alteration, a heterozygous stop gain of BRCA2 (p.K3326X) was detected as constitutive. This variant is recorded in ClinVar as "benign"; however, it is found at low frequency in healthy individuals (ExAc allele frequency $=0.7 \%)$. BRCA2 mRNA expression level was evaluated, but it was found not altered in this case with respect to the other PCC cases (data not shown). Finally, the rare germline variants of $A T R X$ and KTM2D, identified in N56, and of $\mathrm{MDH} 2$, in N53, are missense variants occurring in association with a well-defined somatic mutation on a susceptibility gene (VHL in N56 and RET in N53) and then it is not clear whether they could have a role on the tumor onset.

\section{Discussion}

In this study, we performed whole exome sequencing on a set of twelve PCCs, clinically defined as sporadic, and we found that $50 \%$ of PCCs examined had somatic mutations on the known susceptibility $V H L, N F 1$, and RET genes. In addition to these driver mutations, other somatic passenger mutations were detected. In particular, SYNE1 and ABCC10 were mutated, respectively, in the two RET-mutated cases, while a missense p.G460S mutation of RAD54B affected a NF1 mutated case, suggesting these other events may play a potential role on PCCs pathogenesis and development. Nuclear envelope 1 (SYNE1) gene encodes several different isoforms involved in a variety of cellular processes including cytokinesis, Golgi function, and nuclear organization and structural integrity and positioning of the nucleus $[17,18]$. Mutations of SYNE1 have been found in colorectal cancer, glioblastoma, and ovarian cancer, and methylation of the gene was also frequently found in lung adenocarcinoma and colorectal cancer [19-23]. On the other side, RAD54B is a telomere-related gene involved in DNA repair process, and coding-missense changes of this gene have been found in familial breast cancer cases not explained by mutations in the best-known high susceptibility genes BRCA1 and BRCA2 [24]. These findings may be even more relevant in PCCs, given the highest degree of heritability of this disease. 


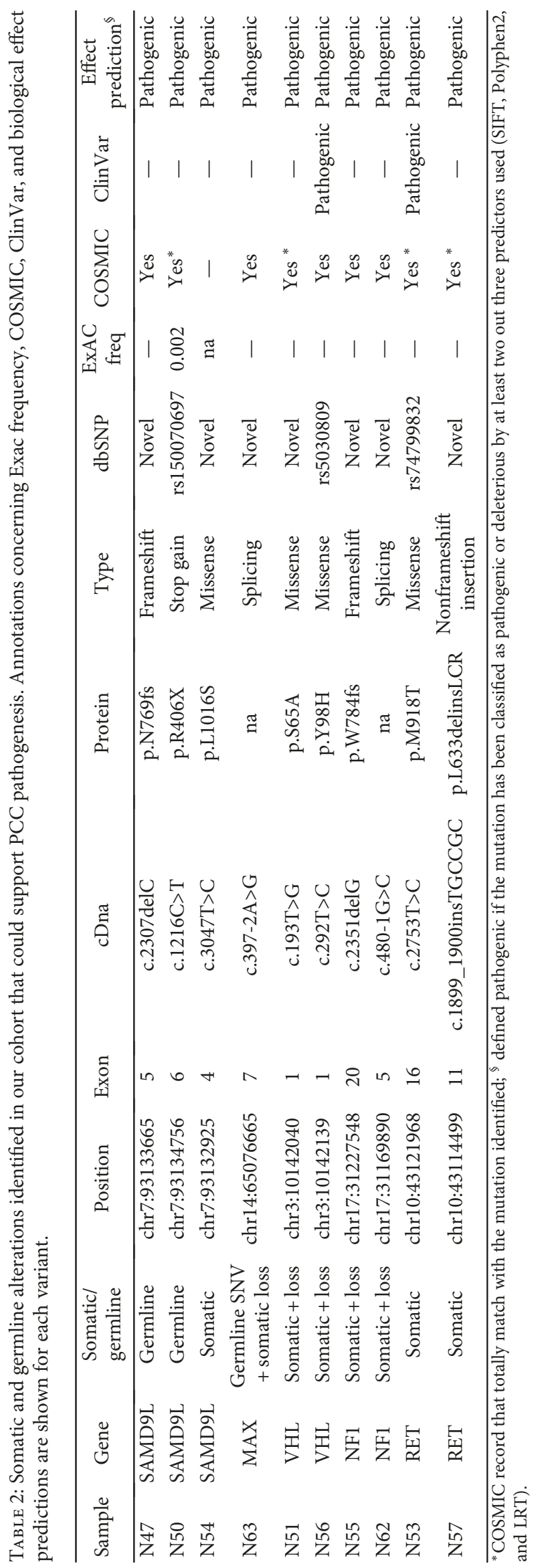




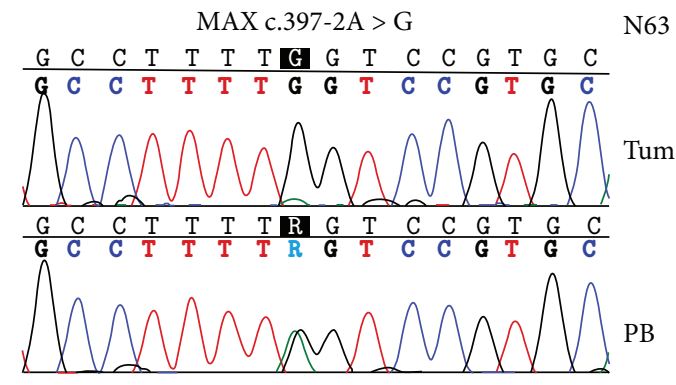

(a)

SAMD9L c.2307delC

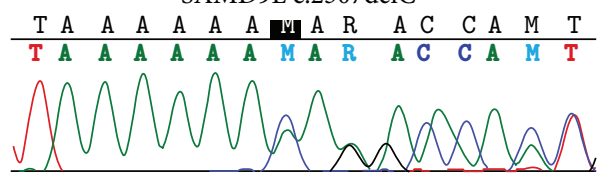

SAMD9L c. $1216 \mathrm{C}>\mathrm{T}$

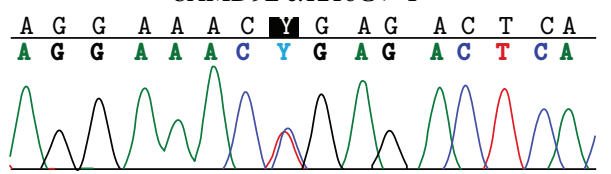

(c)

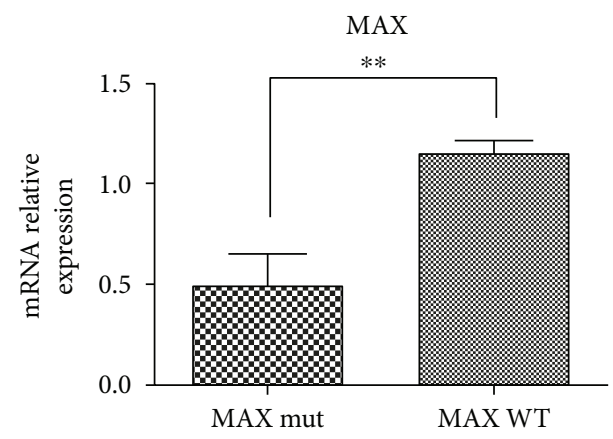

(b)

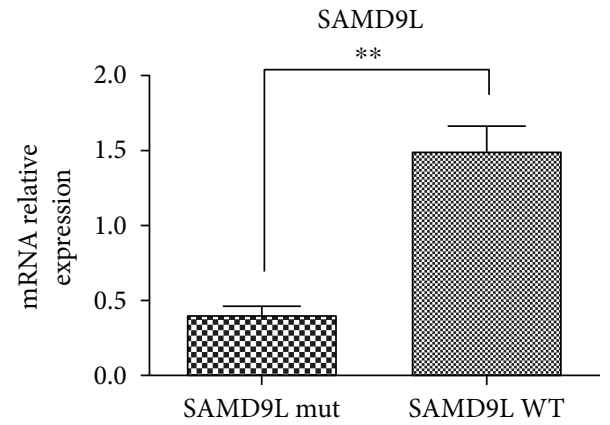

(d)

Figure 2: (a) Loss of heterozygosity of germline MAX mutation detected on N63 tumor. Chromatograms showing homozygous status of c.397-2A $>$ G mutation in the tumor of N63 (upper panel), while in the germline it is heterozygous (lower panel). (b) MAX mRNA relative expression of MAX mutant case (N63) in comparison to the other PCC cases. $P$ value was estimated with unpaired $t$-test $\left({ }^{* *} P<0.01\right)$. (c) Validation of SNV detected on SAMD9L gene in 2 PCC samples. Germline c.2307delC and c.1216C $>$ T heterozygous mutations detected in both tumor and peripheral blood of N47 and N50, respectively. (d) SAMD9L mRNA relative expression of the 2 SAMD9L mutant cases (N47 and N50) in comparison to the other PCC cases. $P$ value was estimated with unpaired $t$-test $\left({ }^{* *} P<0.01\right)$.

Of note on this topic, the identification of germline variants in half of the sporadic PCC samples was analyzed, among which those occurring in MAX and SAMD9L genes may be extremely interesting. Indeed, some reports disclose the importance of screening for germline variants also in sporadic cases, especially on susceptibility genes $[25,26]$, and besides the potential role played in PCCs pathogenesis, the detection of these germline variants in patients clinically defined as sporadic may suggest the existence of unknown multineoplasia hereditary diseases.

In our study, we have identified a variant of MAX (c.397$2 A>G$ ), affecting splice site and pathogenic for the protein function, with the loss of the wild-type allele in the tumor as second hit. Of note, this patient is a young adult of 26 years old, and the presence of a germline variant in a known cancer susceptibility gene may suggest that the PCC in this patient could be the first clinical expression of a hereditary disease still undefined. It is already known that around 1\% of PCC patients were negative for mutations in the other known susceptibility genes carried a germline mutation affecting $M A X[26,27]$. About 20 variants affecting $M A X$ have been already described distributed along the gene, but more frequently involving exons 3 and 4 , matching some of the most important residues within the conserved bHLH-Zip domain of MAX. Most mutations lead to truncated proteins, with the expected $\mathrm{LOH}$ affecting the remaining wild-type allele of the MAX tumor suppressor gene [26, 27]. Two truncating MAX mutations affecting exon $3($ c. $97 \mathrm{C}>\mathrm{T})$ and 4 (c.185_186delA) and three missense variants (c.67G $>A$, c. $281 \mathrm{~T}>\mathrm{C}$, and c.425C $>\mathrm{T}$ ) located in exons 3, 4, and 5, respectively, have been identified [27]. Furthermore, other mutations affecting the initial methionine $(c .2 \mathrm{~T}>\mathrm{A})$, creating a premature stop codon $(\mathrm{c} .25 \mathrm{del}, \mathrm{c} .97 \mathrm{C}>\mathrm{T}, \mathrm{c} .223 \mathrm{C}>\mathrm{T}$, and $c .244 \mathrm{C}>\mathrm{T}$ ) or affecting a donor/acceptor splice site (c.171+1G>A and c.295+1G>T), have been subsequently reported [26]. In addition, 2 deletions were identified: the first caused an inframe loss of 6 highly conserved amino acids within the first helix of the protein (c.140_157del), and the second, spanned the whole gene (c.1-?.483+?del) [26]. The MAX mutant case of our cohort showed a marked downregulation of MAX at mRNA level; however, it did not show a significant modulation of the genes involved in cortical admixture phenotype. Conversely to this finding, two cases of MAX mutant PCC were described to overexpress adrenal cortex markers (including CYP11B2, CYP21A2, and STAR) supporting an association between MAX mutation and the cortical admixture PCC subtype [16]. Further studies on larger cohort will be needed to assess this association.

Moreover, we have found three germline variants in BRCA2 and SAMD9L in other two PCC patients wild-type 
for mutations in susceptibility genes. Specifically, even if it is a rare variant (ExAc allele frequency $=0.7 \%$ ) recently described as associated with an increased risk of developing breast and ovarian cancers [28], the heterozygous stop gain of BRCA2 (p.K3326X) is considered as "benign" in ClinVar. In support of this consideration, we did not detect any variation of BRCA2 mRNA expression level. Thus, we cannot draw any conclusion on the role of this variant and PCC onset. On the contrary, the 2 rare germline variants of SAMD9L detected in two sporadic PCC cases were both producing a premature STOP of the protein (a p.N769fs frameshift deletion and a nonsense p.R406X) and were associated with significant downregulation of SAMD9L mRNA, supporting the pathogenicity of these alterations. The function of this gene is not well characterized; however, evidence has accumulated supporting the role of $S A M D 9 \mathrm{~L}$ in cell proliferation and tumor suppression. In particular, somatic mutations have been found in hepatitis B-related hepatocellular carcinomas [29] and inactivation of SAMD9L has been recently correlated with myeloid transformation [30]. Interestingly, a somatic p.L1016S mutation of SAMD9L was detected in another PCC sample of our cohort, and additionally a p. P636S (COSM3412478) was reported in one case of TCGA PCC/PGL dataset (http://cancergenome. nih.gov/). Taken together, these data could support a possible role of SAMD9L in PCC biology; however, functional studies will be needed to further assess this hypothesis.

\section{Conclusions}

Taken together, the discovery of novel germline variants of known cancer-related genes in sporadic PCC patients, wild-type for mutations in susceptibility genes, may suggest that the likely existence of other multineoplasia syndrome. On the contrary, the role of the other rare germline variants $A T R X, K T M 2 D$, and $M D H 2$ genes identified in two cases having a well-defined somatic mutation on a susceptibility gene (RET or $V H L)$ remains still unclear.

Although the pathogenetic role of all these variants is still not known, due to the large number of susceptibility genes implicated in the diagnosis of inherited PCCs and PGLs, we confirm also what other authors stated that the nextgeneration sequencing technology is ideally suited for carrying out genetic screening of these individuals [31]. Moreover, the high degree of heritability of PCCs and the wide number of germline variants described suggest the likely need of a more extended genetic counselling and the type and duration of the surveillance program of patients affected by PCCs with variants of unknown significance. Finally, these findings may underlie the possible occurrence of novel hereditary diseases that remain still undefined.

In conclusion, additional somatic passenger mutations can be associated to known susceptibility VHL, NF1, and RET genes in PCCs and a wide number of germline variants with still unknown clinical significance can be detected in these patients. Therefore, many efforts should be aimed to better define the pathogenetic role of all these germline variants for discovering novel potential therapeutic targets for this disease still orphan of effective treatments.

\section{Data Availability}

All sequencing data are available upon request to the corresponding author.

\section{Conflicts of Interest}

The authors declare no conflict of interest.

\section{Authors' Contributions}

Milena Urbini and Margherita Nannini contributed equally to this work.

\section{Supplementary Materials}

Supplementary Table 1: list of somatic mutations identified in our cohort. Annotations concerning ExAC frequencies, COSMIC, and effect prediction are shown for each variant. In yellow are highlighted mutations affecting PCC susceptibility genes. Supplementary Table 2: list of rare germline variants identified. Annotations concerning Exac frequency, COSMIC, ClinVar, and biological effect predictions (SIFT, Polyphen2, and LRT) are shown for each variant. (Supplementary Materials)

\section{References}

[1] J. W. M. Lenders, G. Eisenhofer, M. Mannelli, and K. Pacak, "Phaeochromocytoma," Lancet, vol. 366, no. 9486, pp. 665675, 2005.

[2] G. Eisenhofer, S. R. Bornstein, F. M. Brouwers et al., "Malignant pheochromocytoma: current status and initiatives for future progress," Endocrine-Related Cancer, vol. 11, no. 3, pp. 423-436, 2004.

[3] P. L. M. Dahia, "Pheochromocytoma and paraganglioma pathogenesis: learning from genetic heterogeneity," Nature Reviews Cancer, vol. 14, no. 2, pp. 108-119, 2014.

[4] J. Favier, L. Amar, and A. P. Gimenez-Roqueplo, "Paraganglioma and phaeochromocytoma: from genetics to personalized medicine," Nature Reviews. Endocrinology, vol. 11, no. 2, pp. 101-111, 2015.

[5] N. Burnichon, L. Vescovo, L. Amar et al., "Integrative genomic analysis reveals somatic mutations in pheochromocytoma and paraganglioma," Human Molecular Genetics, vol. 20, no. 20, pp. 3974-3985, 2011.

[6] N. Burnichon, A. Buffet, B. Parfait et al., "Somatic NF1 inactivation is a frequent event in sporadic pheochromocytoma," Human Molecular Genetics, vol. 21, no. 26, pp. 5397-5405, 2012.

[7] N. H. Cho, H. W. Lee, S. Y. Lim, S. Kang, W. Y. Jung, and C. S. Park, "Genetic aberrance of sporadic MEN 2A component tumours: analysis of RET," Pathology, vol. 37, no. 1, pp. 10-13, 2005.

[8] I. Comino-Méndez, A. A. de Cubas, C. Bernal et al., "Tumoral EPAS1 (HIF2A) mutations explain sporadic pheochromocytoma and paraganglioma in the absence of erythrocytosis," Human Molecular Genetics, vol. 22, no. 11, pp. 2169-2176, 2013.

[9] J. Crona, A. Delgado Verdugo, R. Maharjan et al., "Somatic mutations in $H$-RAS in sporadic pheochromocytoma and 
paraganglioma identified by exome sequencing," The Journal of Clinical Endocrinology and Metabolism, vol. 98, no. 7, pp. E1266-E1271, 2013.

[10] L. Fishbein, S. Khare, B. Wubbenhorst et al., "Whole-exome sequencing identifies somatic ATRX mutations in pheochromocytomas and paragangliomas," Nature Communications, vol. 6, no. 1, p. 6140, 2015.

[11] R. Hrasćan, N. Pećina-Slaus, T. N. Martić et al., “Analysis of selected genes in neuroendocrine tumours: insulinomas and phaeochromocytomas," Journal of Neuroendocrinology, vol. 20, no. 8, pp. 1015-1022, 2008.

[12] P. Komminoth, E. Kunz, O. Hiort et al., "Detection of RET proto-oncogene point mutations in paraffin-embedded pheochromocytoma specimens by nonradioactive single-strand conformation polymorphism analysis and direct sequencing," The American Journal of Pathology, vol. 145, no. 4, pp. 922929, 1994.

[13] R. A. Toledo, Y. Qin, Z. M. Cheng et al., "Recurrent mutations of chromatin-remodeling genes and kinase receptors in pheochromocytomas and paragangliomas," Clinical Cancer Research, vol. 22, no. 9, pp. 2301-2310, 2016.

[14] Z. Zhuang, C. Yang, F. Lorenzo et al., "Somatic HIF2A gain-offunction mutations in paraganglioma with polycythemia," The New England Journal of Medicine, vol. 367, no. 10, pp. 922930, 2012.

[15] A. Flynn, D. Benn, R. Clifton-Bligh et al., "The genomic landscape of phaeochromocytoma," The Journal of Pathology, vol. 236, no. 1, pp. 78-89, 2015.

[16] L. Fishbein, I. Leshchiner, V. Walter et al., "Comprehensive molecular characterization of pheochromocytoma and paraganglioma," Cancer Cell, vol. 31, no. 2, pp. 181-193, 2017.

[17] J. Fan and K. A. Beck, "A role for the spectrin superfamily member Syne-1 and kinesin II in cytokinesis," Journal of Cell Science, vol. 117, no. 4, pp. 619-629, 2004.

[18] L. L. Gough, J. Fan, S. Chu, S. Winnick, and K. A. Beck, "Golgi localization of Syne-1," Molecular Biology of the Cell, vol. 14, no. 6, pp. 2410-2424, 2003.

[19] M. Tessema, R. Willink, K. Do et al., "Promoter methylation of genes in and around the candidate lung cancer susceptibility locus 6q23-25," Cancer Research, vol. 68, no. 6, pp. 1707$1714,2008$.

[20] K. E. Schuebel, W. Chen, L. Cope et al., "Comparing the DNA hypermethylome with gene mutations in human colorectal cancer," PLoS Genetics, vol. 3, no. 9, pp. 1709-1723, 2007.

[21] T. Sjoblom, S. Jones, L. D. Wood et al., "The consensus coding sequences of human breast and colorectal cancers," Science, vol. 314, no. 5797, pp. 268-274, 2006.

[22] D. L. Masica and R. Karchin, "Correlation of somatic mutation and expression identifies genes important in human glioblastoma progression and survival," Cancer Research, vol. 71, no. 13, pp. $4550-4561,2011$.

[23] J. A. Doherty, M. A. Rossing, K. L. Cushing-Haugen et al., "ESR1/SYNE1 polymorphism and invasive epithelial ovarian cancer risk: an Ovarian Cancer Association Consortium Study," Cancer Epidemiology Biomarkers \& Prevention, vol. 19, no. 1, pp. 245-250, 2010.

[24] K. Yanowsky, A. Barroso, A. Osorio, M. Urioste, J. Benitez, and B. Martinez-Delgado, "Mutational analysis of telomere genes in BRCA1/2-negative breast cancer families with very short telomeres," Breast Cancer Research and Treatment, vol. 134, no. 3, pp. 1337-1343, 2012.

[25] J. Welander, A. Andreasson, C. C. Juhlin et al., "Rare germline mutations identified by targeted next-generation sequencing of susceptibility genes in pheochromocytoma and paraganglioma," The Journal of Clinical Endocrinology and Metabolism, vol. 99, no. 7, pp. E1352-E1360, 2014.

[26] N. Burnichon, A. Cascon, F. Schiavi et al., "MAX mutations cause hereditary and sporadic pheochromocytoma and paraganglioma," Clinical Cancer Research, vol. 18, no. 10, pp. 2828-2837, 2012.

[27] I. Comino-Méndez, F. J. Gracia-Aznárez, F. Schiavi et al., "Exome sequencing identifies MAX mutations as a cause of hereditary pheochromocytoma," Nature Genetics, vol. 43, no. 7, pp. 663-667, 2011.

[28] H. D. Meeks, H. Song, K. Michailidou et al., "BRCA2 Polymorphic Stop Codon K3326X and the Risk of Breast, Prostate, and Ovarian Cancers," Journal of the National Cancer Institute, vol. 108, no. 2, article djv315, 2015.

[29] Q. Wang, Y. Y. Zhai, J. H. Dai, K. Y. Li, Q. Deng, and Z. G. Han, "SAMD9L inactivation promotes cell proliferation via facilitating G1-S transition in hepatitis B virus-associated hepatocellular carcinoma," International Journal of Biological Sciences, vol. 10, no. 8, pp. 807-816, 2014.

[30] A. Nagamachi, H. Matsui, H. Asou et al., "Haploinsufficiency of SAMD9L, an endosome fusion facilitator, causes myeloid malignancies in mice mimicking human diseases with monosomy 7," Cancer Cell, vol. 24, no. 3, pp. 305-317, 2013.

[31] NGS in PPGL (NGSnPPGL) Study Group, R. A. Toledo, N. Burnichon et al., "Consensus statement on nextgeneration-sequencing-based diagnostic testing of hereditary phaeochromocytomas and paragangliomas," Nature Reviews Endocrinology, vol. 13, no. 4, pp. 233-247, 2017. 


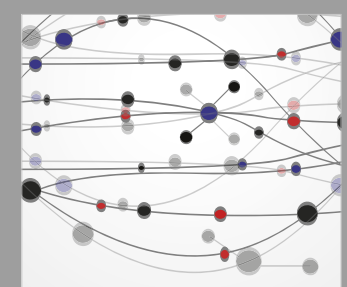

The Scientific World Journal
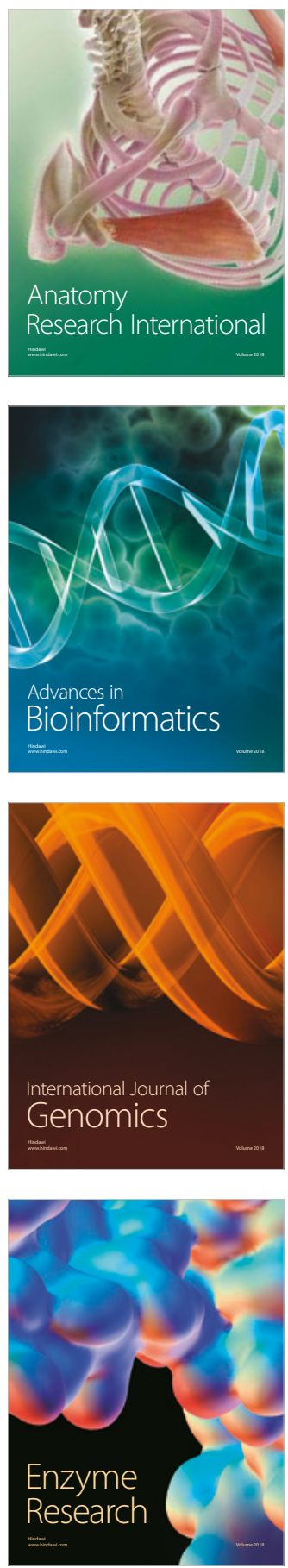
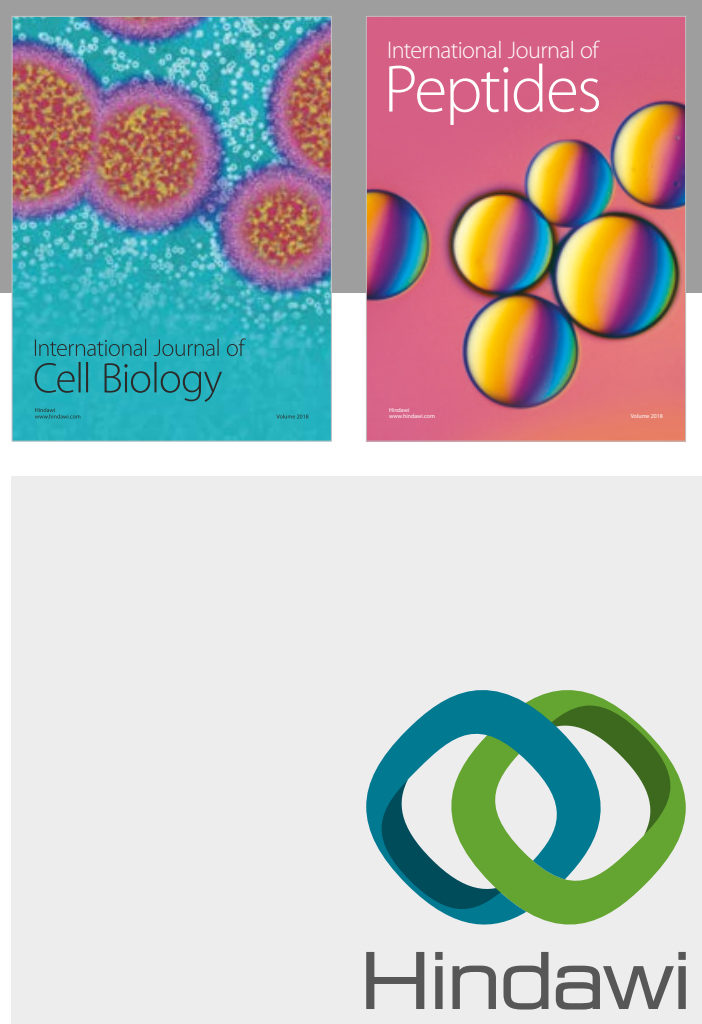

Submit your manuscripts at

www.hindawi.com
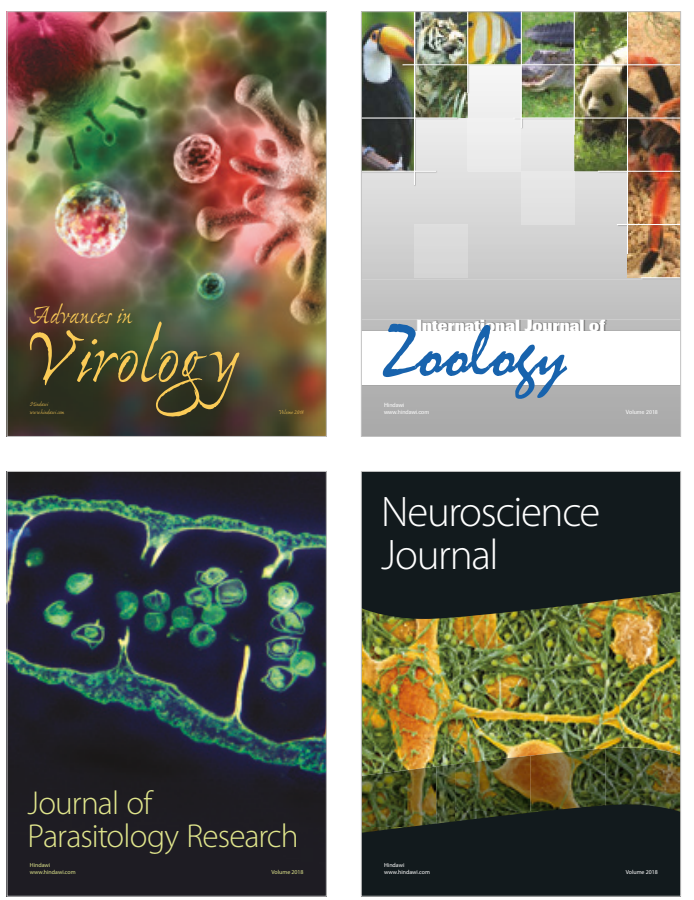
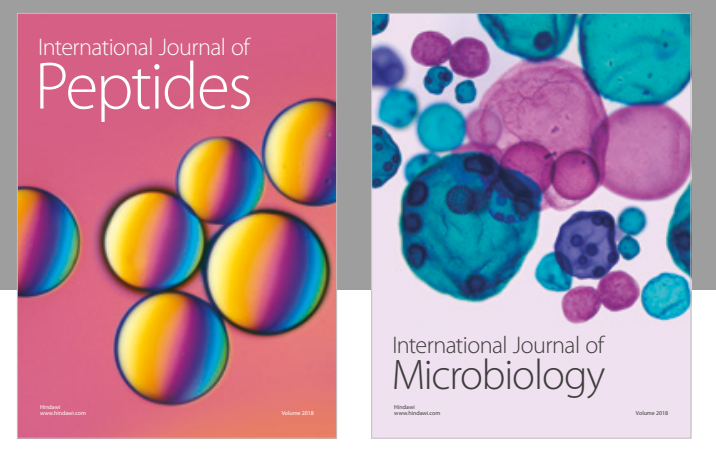

nternational Journal of Microbiology
Journal of
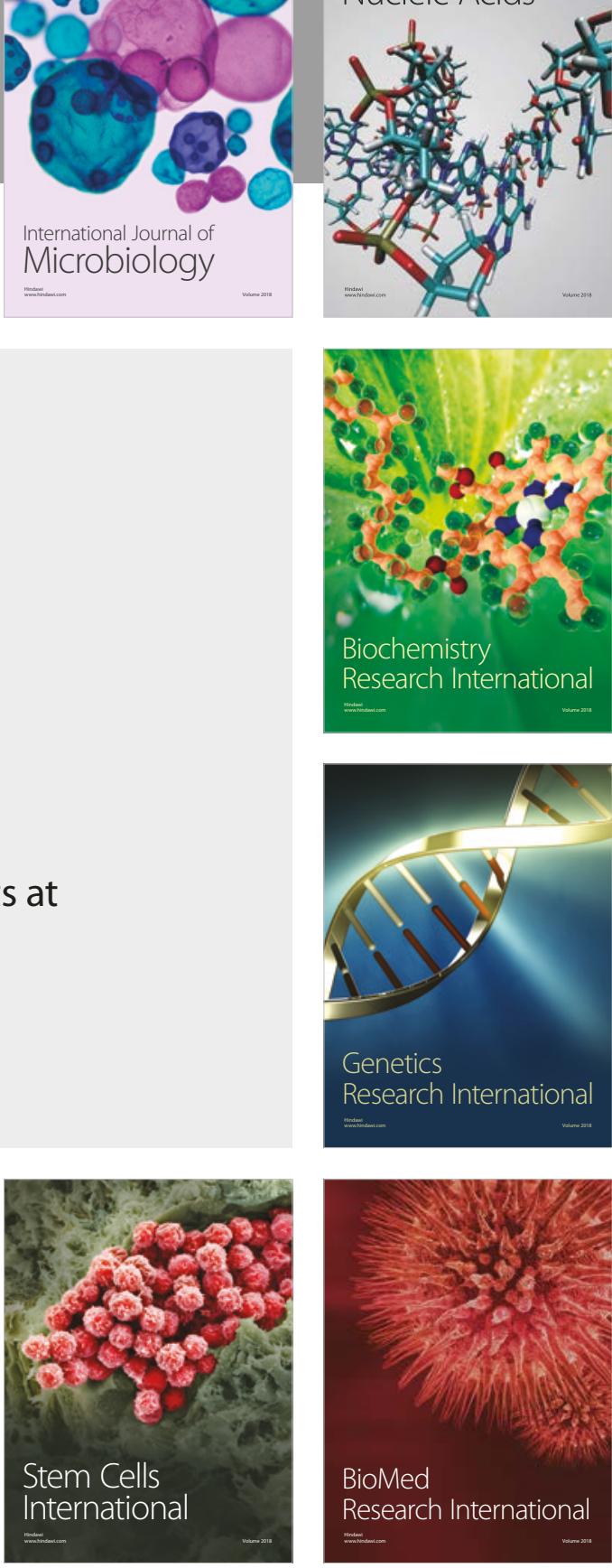
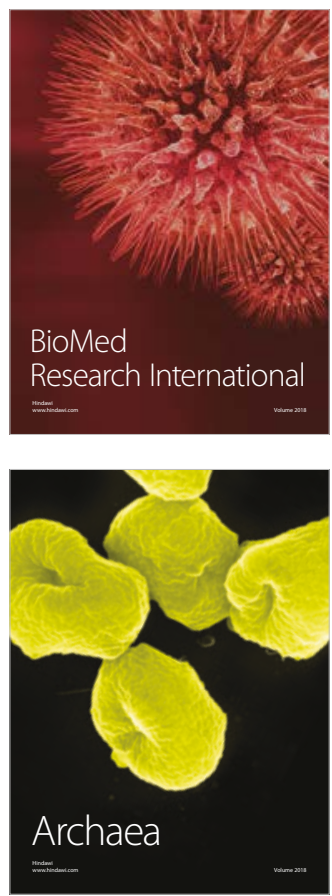